\title{
The role of kisspeptin on aromatase expression in breast cancer
}

\author{
Yilmaz $\mathrm{MB}^{1}$, Oksuz $\mathrm{H}^{1}$, Ilgaz $\mathrm{NS}^{1}$, Ocal I², Norizadeh Tazehkand $\mathrm{M}^{3}$ \\ Department of Medical Biology, Faculty of Medicine, Cukurova University, Adana, Turkey. \\ mehmetbertanyilmaz@gmail.com
}

\begin{abstract}
AIM: Kisspeptin is a reproductive peptide hormone that has anti-metastatic roles in several cancer types including colon, lung, and brain cancer. However, in breast cancer, increasing of kisspeptin expression induces aggressiveness of tumors, which in turn exacerbates breast cancer prognosis.

MATERIAL AND METHODS: Breast cancer cell lines MCF7 and SKBR3 were cultured in MEM (phenol red free) containing $10 \%$ fetal bovine. Treatments were performed, at $70 \%$ confluency, after 24-hour serum deprivation in serum free medium for 6, 24 and 48 hours. Aromatase (CYP19A1) and kisspeptin receptor (GPR54) mRNA expression were determined by real time Taqman Assay.

RESULT: Kisspeptin induced aromatase (CYP19A1) and kisspeptin receptor (GPR54) mRNA expression, while this induction was abolished by kisspeptin receptor inhibitor in MCF7 cells. In SKBR3 cells, however, even though there was an increase in GPR54 mRNA expression with kisspeptin, the induction of CYP19A1 was not observed. CONCLUSION: The inducing effect of kisspeptin on aromatase expression is possibly mediated via kisspeptin receptor and estrogen receptor dependent mechanisms (Fig. 5, Ref. 27). Text in PDF www.elis.sk. KEY WORDS: breast cancer, aromatase, kisspeptin, metastin, kisspeptin receptor.
\end{abstract}

\section{Introduction}

Sex steroids play active roles in shaping neural functions and reproductive behavior, while regulating cellular organization and mammalian development in various ways. The synthesis of sex steroids begins with the entry of cholesterol into the mitochondria followed by the formation of estrogen, progesterone, and testosterone as the result of the six basic enzymatic steps completed in endoplasmic reticulum. Estrogen can be synthesized by various tissues and organs such as: gonads, adrenal cortex, and brain and locally in breast. Aromatase-catalyzed conversion of $\mathrm{C}_{19}$ steroids to estradiol $\left(\mathrm{E}_{2}\right)$, which is biologically active estrogen, is the rate limiting step of estrogen biosynthesis $(1,2)$.

In humans, aromatase is encoded by a single copy gene CYP19A1. CYP19A1 gene is located on the long arm of chromosome 15 , with a $30 \mathrm{~kb}$ coding region and a $95 \mathrm{~kb}$ regulatory promoter region, total $125 \mathrm{~kb}$ in length. The aromatase protein consists of nine exons (II-X), and on the 5'-upstream of exon II, where different primary exons are located with number of different promot-

${ }^{1}$ Department of Medical Biology, Faculty of Medicine, Cukurova University, Adana, Turkey, ${ }^{2}$ Department of Biophysics, Faculty of Medicine, Cukurova University, Adana, Turkey, and ${ }^{3}$ Department of Pharmaceutical Biotechnology, Faculty of Pharmacy, Bulent Ecevit University, Zonguldak, Turkey

Address for correspondence: M.B. Yilmaz, Cukurova University, Faculty of Medicine, Department of Medical Biology, Adana, Turkey.

Acknowledgments: This study was supported by grants from Cukurova University Scientific Research Support Program project numbers TSA2015-3255 and TSA-2016-6911. ers that direct the tissue-specific alternative transcription of these primary exons. This gene organization leads to the formation of different 5'-untranslated region-containing aromatase mRNAs, but since these regions are not involved in the aromatase protein structure, the protein product formed by the translation of these different mRNAs, is identical in all tissue types (Fig. 1) $(3,4)$.

The physiological role of aromatase has been understood with studies on aromatase gene-deficient (ArKO) mice and men with mutations causing an inactive aromatase gene. In women, the highest aromatase expression is observed in the ovary in premenopausal period and in adipose tissue in postmenopausal period. The cytokines, such as: tumor necrosis factor and interleukin 11 (IL-11), release from malignant breast epithelial cells, suppress adipogenic transcription factors and prevent the transformation of fibroblasts into mature adipocyte cells. This phenomenon was observed in the fibroblast cells of breast tissue, which provide a structural support to adipose tissue leading tumor growth and development. Estrogen produced by undifferentiated fibroblasts due to high levels of aromatase causes mitogenic effects on malignant epithelial cells, thereby contributing to the spread of malignant cells by increasing cellular growth and proliferation rates in breast cancer (5). It is known that malignant epithelial cells synthesize and secrete many yet undefined factors that increase aromatase expression $(6,7,8)$.

Kisspeptin has been also reported in several aspects of cancer, either in a causative or preventive manner. Even though kisspeptin has been first introduced as an anticancerogenic agent, which was defined as metastin, when first discovered, now it is commonly known to be involved in cancer formation and aggression in most peripheral tissue types. Kisspeptines are a family of neuropep- 


\section{CYP19A1 (aromatase) GENE}

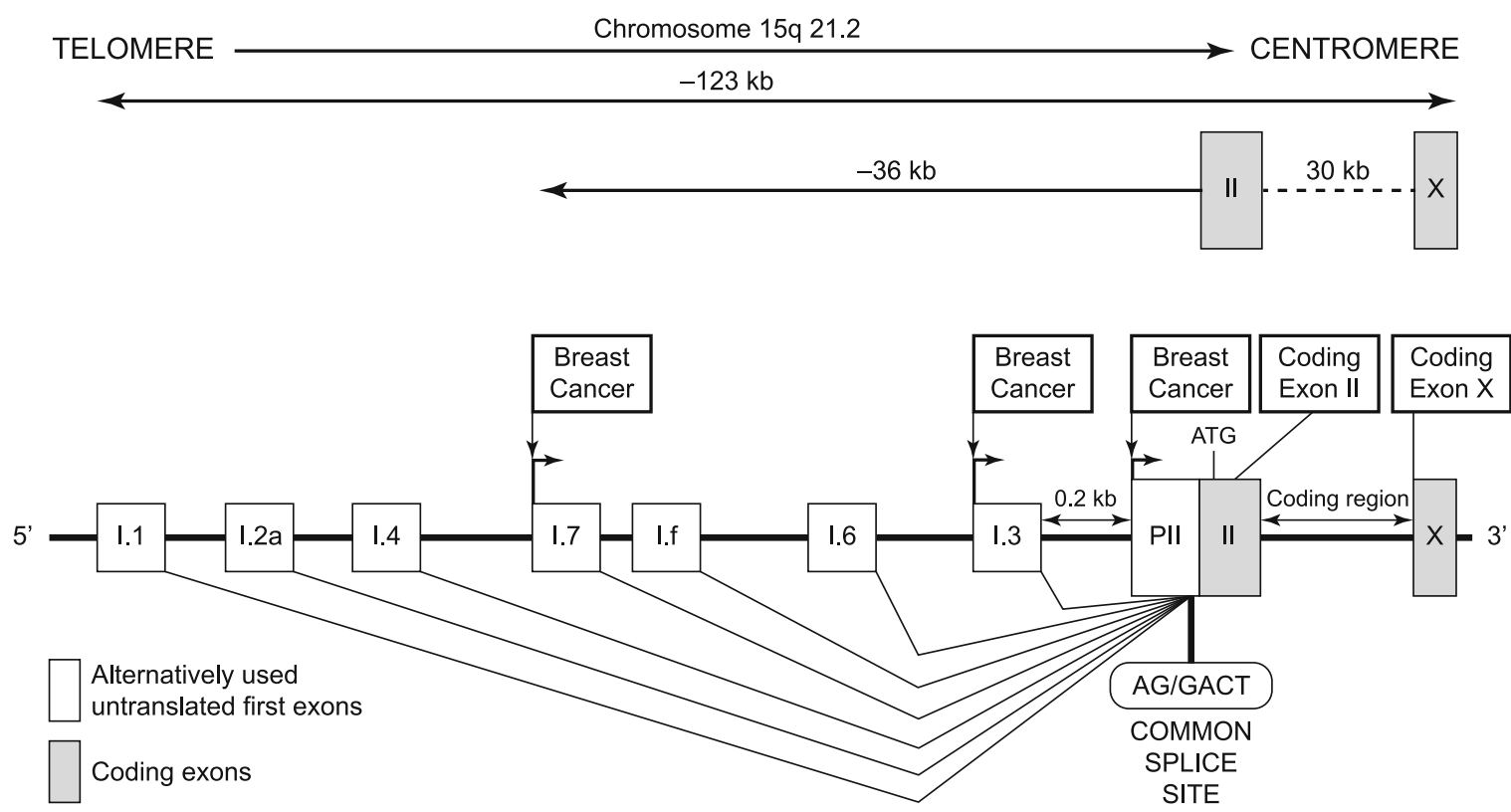

Fig. 1. Aromatase (CYP19) gene. Expression of the aromatase gene in breast is regulated by the tissue-specific activation of a number of promoters via alternative splicing, which are involved in breast cancer formation to some extent.

tides encoded by Kiss 1 gene, which has three exons located in the long arm of the first chromosome. Recent studies report the importance of Kisspeptin in gonadotropin secretion by showing that kisspeptin is a regulatory element for the release of $\mathrm{GnRH}$ in neurons. Kisspeptines also have roles in sexual differentiation of the brain, timing of puberty, and in adults, control of gonadotropin secretion via feedback mechanisms by gonadal hormones and control of fertility by metabolic and environmental (eg, photoperiod) cues $(9,10,11)$.

The biological features mentioned above suggest that kisspeptins have important pathophysiological and therapeutical roles in diverse fields beyond their reproduction-related physiological roles in the brain and of these roles, the most pronounced ones, involves cancer. In breast cancer, tumors kisspeptin mRNA and protein expression increase further, which is found to be associated with a poor patient diagnosis. Kisspeptin also induces invasiveness and decreases adhesiveness of human breast cancer cells. Interestingly, kisspeptin was also found to be associated with genes or proteins that regulate cell cycle, proliferation or invasiveness such as: ESR1, HER2, VEGF or P53 $(11,12,13)$. These, ESR1 and P53 were found to be directly associated with aromatase promoter $(5,14,15)$. In this regard, it is possible that kisspeptin exerts its effects on breast cells through aromatase. In this study, we used two different cell lines MCF7 and SKBR3, former is ESR1 positive, and the latter is ESR1 negative, both of which express KISS1, GPR54 and CYP19A1 $(16,17)$. In summary, we wanted to determine whether aromatase was the one of the mediators of kisspeptin action in breast cancer.

\section{Materials and methods}

\section{Cell culture}

Breast cancer cell lines MCF7 and SKBR3 were cultured in MEM (phenol red free) containing 10\% fetal bovine serum (FBS) (Gibco, Grand Island NY, USA) and 1\% penicillin-streptomycin at $37{ }^{\circ} \mathrm{C}$ and $5 \% \mathrm{CO}_{2}$. Treatments were performed, at $70 \%$ confluency, after 24-hour serum deprivation in serum free medium for 6, 24 and 48 hours.

RNA isolation and quantitative Real-Time PCR: Total RNA was isolated using the TRIzol reagent from the cells (Invitrogen, Carlsbad CA, USA). DNAase cleavage was performed in the column by using DNase I Kit. (Qiagen, Valencia, USA). The integrity of the isolated RNA was confirmed by running $5 \mu \mathrm{g}$ of RNA on 1 $\%$ formaldehyde gel. cDNA was synthesized using SuperScript $\mathrm{t}^{\mathrm{TM}}$ III First-Strand kit oligonucleotide (dT) primers as recommended by the supplier (Invitrogen). Real-Time PCR was performed with the ABI 7900HT Fast Real-Time PCR System (Applied Biosystems) using the Taqman PCR/Probe Assays (Applied Biosystems, Foster City, USA) by using two microliters of cDNA.(Primers used: Cyp19a1:Mm00484049_m1 Gpr54:Mm00475046_m1 and GAPDH: Mm99999915_g1).

Polymerase chain reaction was carried out by using Taqman gene expression mix in a total volume $25 \mu \mathrm{l}$ in the form of three replicates. Reaction was incubated at $50{ }^{\circ} \mathrm{C}$ for $2 \mathrm{~min}$ and then at $95^{\circ} \mathrm{C}$ for $10 \mathrm{~min}$ following 40 cycles at $95{ }^{\circ} \mathrm{C}$ for $15 \mathrm{~s}$ and then at $60{ }^{\circ} \mathrm{C}$ for $1 \mathrm{~min}$. The threshold cycling value (CT) in the studies; the cycle, in which the amount of fluorescence in the reaction 
$776-780$

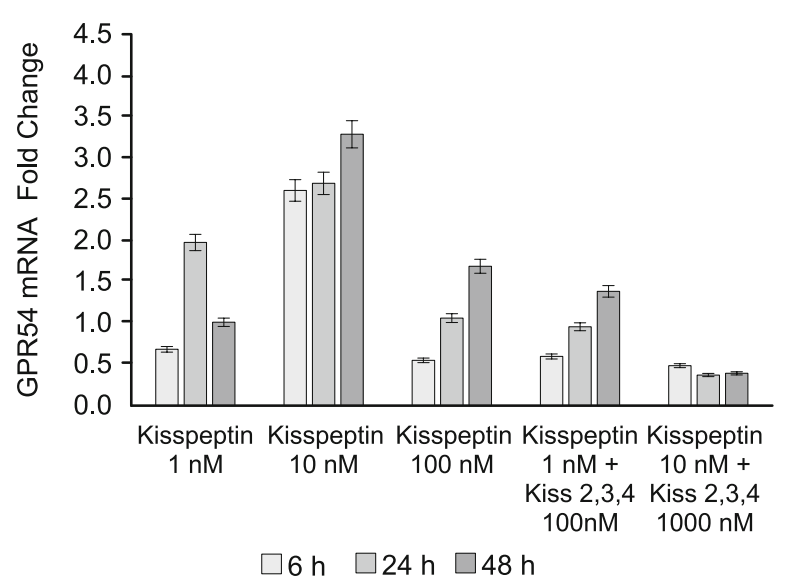

Fig. 2. Kisspeptin receptor (GPR54) mRNA expression in the MCF7 cancer cell line. Fold change of GPR54 mRNA expression of various treatment regime is shown. Results were normalized to the expression levels of GAPDH gene as an endogenous control. The results are expressed as the mean \pm S.E.M. from three independent experiments $(\mathrm{p}<0.01$, paired $\mathrm{t}$ test).

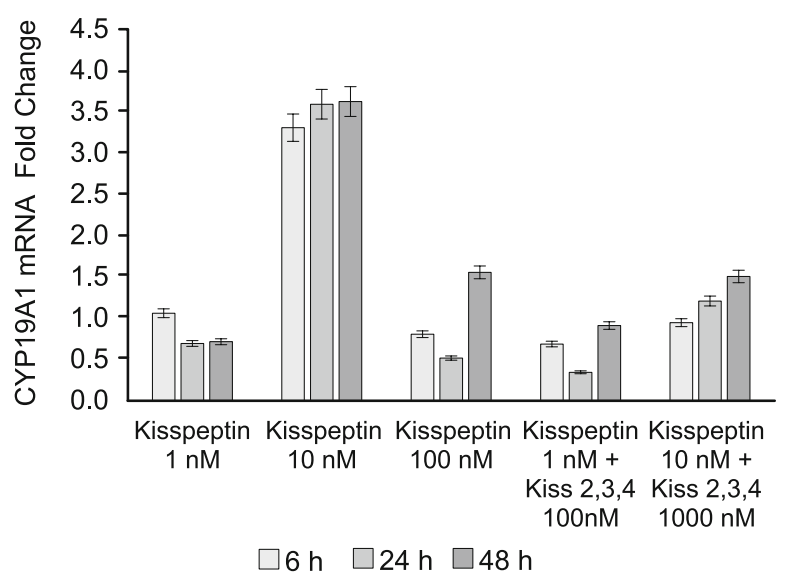

Fig. 3. Aromatase (CYP19A1) mRNA expression in the MCF7 cancer cell line. Fold change of CYP19A1 mRNA expression of various treatment regime is shown. Results were normalized to the expression levels of GAPDH gene as an endogenous control. The results are expressed as the mean \pm S.E.M. from three independent experiments $(\mathrm{p}<0.01$, paired $\mathrm{t}$ test).

medium passed the fixed threshold value was used as the fractional expression. Then the CT values were converted to absolute copy numbers. All cDNA samples were normalized using the GAPDH endogenous control. Genomic DNA presence and reaction specificity were checked by using the "template-free" and "enzymefree" techniques. Three independent experiments were performed to demonstrate repeatability. The specificity of RT-PCR products was confirmed by melt curve analysis, and gel electrophoresis.

Statistical Analysis: StatView 5.0 Statistic Program package (SAS Institute, Cary NC, USA) was used to perform Tukey multiple comparisons test and following welch paired t-test and oneway ANOVA statistical analysis. Significance was accepted as $\alpha$ $=0.05$ and $\beta=0.20$.
Results

In MCF7 cells (ESR1 Positive), Kisspeptin at $1 \mathrm{nM}$ concentration slightly induced kisspeptin receptor expression, but not aromatase expression at $6 \mathrm{~h}$. However, $10 \mathrm{nM}$ kisspeptin induced both kisspeptin receptor and aromatase expression at each time point tested (6, 24 and 48 hours). Kisspeptin receptor and aromatase induction was abolished by Kisspeptin inhibitor K234. At $100 \mathrm{nM}$ kisspeptin concentration, both kisspeptin receptor and aromatase expression was induced at 48h treatment (Figs 2 and 3). In SKBR3 (ESR1 Negative) cells, however, even though kisspeptin induced kisspeptin receptor expression at $10 \mathrm{nM}$, this was also abolished by kisspeptin inhibitor. Kisspeptin did not induce aromatase expression at any concentration and time (Figs 4 and 5).

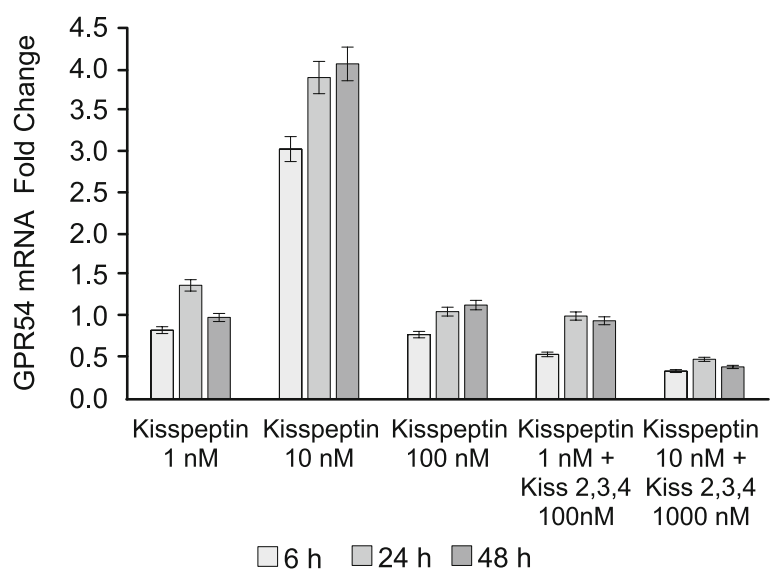

Fig. 4. Kisspeptin receptor (GPR54) mRNA expression in the SKBR3 cancer cell line. Fold change of GPR54 mRNA expression of various treatment regime is shown. Results were normalized to the expression levels of GAPDH gene as an endogenous control. The results are expressed as the mean \pm S.E.M. from three independent experiments (p $<0.01$, paired t test).

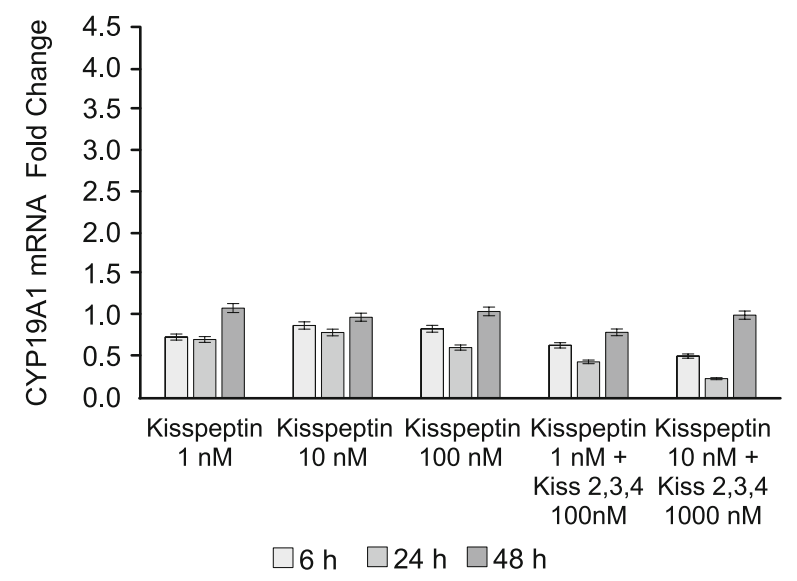

Fig. 5. Aromatase (CYP19A1) mRNA expression in the SKBR3 cancer cell line. Fold change of CYP19A1 mRNA expression of various treatment regime is shown. Results were normalized to the expression levels of GAPDH gene as an endogenous control. The results are expressed as the mean \pm S.E.M. from three independent experiments $(p<0.01$, paired $t$ test). 


\section{Discussion}

In this present study, we uncovered a novel finding, which is the kisspeptin dependent regulation of aromatase in breast adenocarcinoma MCF7 (ESR1 Positive) and SKBR3 (ESR1 Negative) cell lines. Aromatase, which is responsible for the key step in estrogen synthesis, has long been implicated in most aspects of breast cancer (3). Aromatase is expressed in several tissues, including adipose tissue of breast, where its expression levels drastically increase, especially in breast cancer. Current approaches to treat breast cancer introduce aromatase inhibitors, which reduces the local estrogen production (18). An unresolved key question is how aromatase is induced to produce more estrogen in breast cancer.

Epidemiologic studies suggest that circulating estrogen levels initiate tumor formation, however, for tumor growth, sustained estrogen production is required (19). Since the circulating estrogen levels become steady at certain points, there should be some endogenous estrogen producing mechanisms to support and maintain tumor growth locally.

In breast cancer, aromatase is upregulated via various pathways. As mentioned earlier, alternatively used aromatase promoters participate in different regulatory pathways. Of these promoters I1, I3, I4, I7 and PII drive aromatase expression in breast, where a distinct set of transcription factors regulate promoter in a signaling pathway and tissue-specific manner (3). Since many alternative exons are implicated in breast tissue, there is a great plethora of transcription factors reported in breast tumor cells. Aromatase induction in breast cancer mostly functions through exons I3/PII $(4,20)$. Interestingly, these transcription factors are mostly derived from ESR1 dependent signaling pathways $(21,22)$. Estrogen Receptor alpha is also activated by kisspeptin/kisspeptin receptor dependent pathways $(23,24)$. Likewise, in our studies, kisspeptin induced aromatase expression in ESR1 positive MCF7 cells, but not in ESR1 negative SKBR cells. Since kisspeptin has been shown to induce ESR1 (25), and ESR1 can induce CYP19A1 expression $(26,27)$, the result of this research shows that ESR1 is one of the mediators of kisspeptin specifically on aromatase expression.

Therefore, it is plausible to think that kisspeptin-dependent aromatase induction may be mediated through ESR1 dependent pathways, which can induce endogenous aromatase expression. However, there are certain points that need to be clarified in our experiments. One of them is the reason why $1 \mathrm{nM}$ kisspeptin induced kisspeptin receptor, but not aromatase. This finding may be partially due to insufficient kisspeptin receptor induction. Another point to be considered is that kisspeptin receptor repression rate with kisspeptin receptor inhibitor was reduced to a lower extent compared to aromatase levels, which may stem from the fact that kisspeptin acts first before its inhibitor. In our study, aromatase enzyme activity or kisspeptin receptor expression could have been assessed to evaluate their activity or expression, respectively.

In conclusion, the finding of this study showed that kisspeptin induced aromatase possibly via ESR1 dependent pathways. As for future directions, steps involving kisspeptin dependent signaling pathways and their physical association with ESR1 can be investigated.

\section{References}

1. Behan LA, Amir E, Casper RF. Aromatase inhibitors for prevention of breast cancer in postmenopausal women: A narrative review. Menopause $2015 ; 22: 342-350$.

2. Bulun SE, Lin Z, Imir G, Amin S, Demura M, Yilmaz B et al. Regulation of aromatase expression in estrogen-responsive breast and uterine disease: From bench to treatment. Pharmacol Rev 2005; 57: 359-83.

3. Castellano JM, Roa J, Luque RM, Dieguez C, Aguilar E, Pinilla L et al. Kiss-1/kisspeptins and the metabolic control of reproduction: Physiologic roles and putative physiopathological implications. Peptides 2009; 30: 139-145.

4. Catalano S1, Barone I, Giordano C, Rizza P, Qi H, Gu G et al. Rapid estradiol/ERalpha signaling enhances aromatase enzymatic activity in breast cancer cells. Mol Endocrinol 2009; 23(10): 1634-1645.

5. Clyne CD, Speed CJ, Zhou J, Simpson ER. Liver receptor homologue-1 (LRH-1) regulates expression of aromatase in preadipocytes. J Biol Chem 2002; 277: 20591-20597.

6. Cvetkovic D, Babwah AV, Bhattacharya M. Kisspeptin/KISS1R System in Breast Cancer. J Cancer 2013a; 4: 653-661.

7. Cvetkovic D, Dragan M, Leith SJ, Mir ZM, Leong HS, Pampillo $\mathbf{M}$ et al. KISS1R induces invasiveness of estrogen receptor-negative human mammary epithelial and breast cancer cells. Endocrinology 2013b; 154: 1999-2014.

8. Hevir N1, Trost N, Debeljak N, Rižner TL. Expression of estrogen and progesterone receptors and estrogen metabolizing enzymes in different breast cancer cell lines. Chem Biol Interact 2010; 191 (1-3): 206-216.

9. Jiang Q, He M, Ko WK, Wong AO. Kisspeptin induction of somatolactin- $\alpha$ release in goldfish pituitary cells: Functional role of cAMP/ PKA-, PLC/PKC- and Ca(2+)/calmodulin-dependent cascades. Am J Physiol Endocrinol Metab 2014; 307: E872-884.

10. Jones ME, Boon WC, Proietto J, Simpson ER. Of mice and men: The evolving phenotype of aromatase deficiency. Trends Endocrinol Metab 2006; 17: 55-64.

11. Murata Y, Robertson KM, Jones ME, Simpson ER. Effect of estrogen deficiency in the male: The ArKO mouse model. Mol Cell Endocrinol 2002; 193: 7-12.

12. Pasquier J, Kamech N, Lafont AG, Vaudry H, Rousseau K, Dufour S. Molecular evolution of GPCRs: Kisspeptin/kisspeptin receptors. J Mol Endocrinol 2004; 52: T101-117.

13. Roa J, Aguilar E, Dieguez C, Pinilla L, Tena-Sempere M. New frontiers in kisspeptin/GPR54 physiology as fundamental gatekeepers of reproductive function. Front Neuroendocrinol 2008; 29: 48-69.

14. Samarajeewa NU, Docanto MM, Simpson ER, Brown KA. CREB-regulated transcription co-activator family stimulates promoter II-driven aromatase expression in preadipocytes. Horm Cancer 2013; 4: $233-241$.

15. Taylor J, Pampillo M, Bhattacharya M, Babwah AV. Kisspeptin/ KISS1R signaling potentiates extravillous trophoblast adhesion to typeI collagen in a PKC- and ERK1/2-dependent manner. Mol Reprod Dev 2014; 81: 42-54.

16. Teng Y1, Liu M, Cowell JK. Functional interrelationship between the WASF3 and KISS1 metastasis-associated genes in breast cancer cells. Int J Cancer 2011; 15; 129 (12): 2825-2835. 


\section{6-780}

17. Terasaka T1, Otsuka F, Tsukamoto N, Nakamura E, Inagaki K, Toma K et al. Mutual interaction of kisspeptin, estrogen and bone morphogenetic protein-activity in GnRH regulation by GT1-7 cells. Mol Cell Endocrinol 2013; 381 (1-2): 8-15.

18. Turgeon JL, Carr MC, Maki PM, Mendelson ME, Wise PM. Complex actions of sex steroids in adipose tissue, the cardiovascular system, and brain: Insights from basic science and clinical studies. Endocr Rev 2006; 27: 575-605.

19. Wang X, Docanto MM, Sasano H, Simpson ER, Brown KA. Prostaglandin E2 inhibits p53 in human breast adipose stromal cells: A novel mechanism for the regulation of aromatase in obesity and breast cancer. Cancer Res 2015a; 75: 645-655.

20. Wang X, Simpson ER, Brown KA. p53: Protection against Tumor Growth beyond Effects on Cell Cycle and Apoptosis. Cancer Res 2015b; 75: 5001-5007.

21. Wrobel B, Karasek M. Human sexuality and sex steroids. Neuro Endocrinol Lett 2008; 29: 3-10.
22. Yilmaz MB, Wolfe A, Cheng YH, Glidewell-Kenney C, Jameson JL, Bulun SE. Aromatase promoter I.f is regulated by estrogen receptor alpha (ESR1) in mouse hypothalamic neuronal cell lines. Biol Reprod 2009; 81 (5): 956-965.

23. Yilmaz MB, Zhao H, Brooks DC, Fenkci IV, Imir-Yenicesu G, Attar E et al. Estrogen receptor alpha (Esr1) regulates aromatase (Cyp19a1) expression in the mouse brain. Neuro Endocrinol Lett 2015; 36 (2): 178-182.

24. Zhao H, Innes J, Brooks DC, Reierstad S, Yilmaz MB, Lin Z et al. A novel promoter controls Cyp19a1 gene expression in mouse adipose tissue. Reprod Biol Endocrinol 2009; 7: 37.

25. Zhao Y, Agarwal VR, Mendelson CR, Simpson ER. Estrogen biosynthesis proximal to a breast tumor is stimulated by PGE2 via cyclic AMP, leading to activation of promoter II of the CYP19 (aromatase) gene. Endocrinology 1996; 137: 5739-5742.

26. Ziegler RG, Fuhrman BJ, Moore SC, Matthews CE. Epidemiologic studies of estrogen metabolism and breast cancer. Steroids 2015; 99: 67-75.

27. Zirilli L, Rochira V, Diazzi C, Caffagni G, Carani C. Human models of aromatase deficiency. J Steroid Biochem Mol Biol 2008; 109: 212-218.

Received August 11, 2018. Accepted September 19, 2018. 